\title{
Lifetime Prediction method of LED Light-emitting Device Based on Approximate Analysis
}

\author{
CAI Wenting ${ }^{1}$, LI Sheng ${ }^{2}$, MENG Qinghui ${ }^{2}$, HUANG Haisong ${ }^{2}$ \\ ${ }^{1}$ Faculty of Materials Metallurgy and Chemistry and Devices of Jiangxi Province, Jiangxi University of Science and Technology \\ ${ }^{2}$ School of Science, Jiangxi University of Science and Technology, Jiangxi, Ganzhou 341000
}

\begin{abstract}
Compared with other light sources, LED light source has a longer service life and will not suddenly fail. Due to its high efficiency, energy saving and long life, LED has become the most popular light source at present. However, it is no longer considered to meet the application requirements, and the time that the light flux decays to this level is considered to be the life of the LED. This paper introduces the approximate method used to predict the lumen maintenance life of LED lamps. The experimental results obtained by the approximate method are compared with the TM-21 standard. Eventually, it is concluded that the approximate method provides more reliability information than the original TM-21 standard, and the obtained results are more reference, more accurate and more reliable.
\end{abstract}

\section{Introduction}

In recent years, solid-state lighting has been widely used in various fields of industry and life. As a new generation of green light sources, light-emitting diodes (LEDs) have high efficiency, long life, high stability, relatively environmentally friendly (they do not contain mercury) and power consumption. The low-level advantages have opened up a new revolution in the field of lighting. ${ }^{[1]}$ With the gradual expansion of the LED lamp application market, LED has become one of the most important lighting solutions in the current era, and the market demand for LED lighting is energy-efficient and durable. Therefore, how to accurately predict the life of LED lamps has gradually attracted widespread attention. In general, the lumen maintenance life $\left(L_{p}\right)$ is the most suitable characteristic to measure the performance, life and reliability of LED lighting, ${ }^{[2]}$ and most LED manufacturers currently establish life prediction analysis based on $L_{70}$. Generally speaking, LED life refers to the $L_{70}$ life of general lighting. ${ }^{[3]} L_{70}$ is calculated based on the light output degradation time, which represents the time when the LED lumen output drops from the initial value to $70 \%$ (or the time when the lumen maintains a $30 \%$ drop). Based on the lumens data of 6000 hours or more, this article uses an approximate method to predict the life of the LED. Firstly, use the NLS regression method to perform curve fitting, predict the lumen maintenance life of the LED according to the curve equation obtained by the fitting. Then, use the approximation method to process the data obtained by the fitting, carry out the analysis and evaluation of the reliability of the LED. Therefore, compare and discuss with approximate the evaluation results of the method are compared and discussed the evaluation results of the approximation method with the TM-21 standard prediction method. ${ }^{[4]}$

\section{Basic method theory}

\subsection{NLS regression method}

The NLS method is a parameter estimation method that estimates the parameters of a nonlinear static model based on the minimum sum of squares of errors ${ }^{[5]}$. The function of the nonlinear combination of the model parameters depends on one or more independent variables. Among them, the observation data is obtained through function modelling, and the data is fitted through successive approximation methods. The specific steps of the NLS regression method are as follows:

1) Normalize the luminous flux data of the LED and select the average value to obtain the lumen maintenance data of the sample.

2) Using the NLS regression method, according to formula (1) to curve-fit the lumen maintenance data of the LED.A subsubsection. The paragraph text follows on from the subsubsection heading but should not be in italic.

$$
\varphi(x)=\beta \exp (-\alpha t)
$$

Among them, $\varphi(x)$ is the normalized average of the luminous flux at time $\mathrm{t}$, in other word, the lumen maintenance rate. $\alpha, \beta$ are all curve fitting coefficients, which are constants.

3) Perform logarithmic transformation of equation (1) to obtain the LED lifetime prediction model:

\footnotetext{
${ }^{a}$ Corresponding author: 1320181521@mail.jxust.edu.cn
} 


$$
L_{p}=\frac{\ln \left(\frac{\beta}{P / 100}\right)}{\alpha}
$$

Among them, $L p$ is the lifetime when the lumen of the LED maintains and decays to $\mathrm{P} \%$ of the initial value ( $\mathrm{P}=70$ is adopted in this study).

\subsection{Approximation}

The approximation method predicts the failure time of each unit based on the general degradation model, and projects the "pseudo" failure time when the degradation path reaches the critical failure threshold $\mathrm{D}_{\mathrm{f} .}{ }^{[6]}$ For the general degradation path model, assume that the random sample size is, and the measurement time is $t_{1}, t_{2}, t_{3} \ldots t_{n}$. The performance measurement of the $i$-th unit in the $j$-th test is called $y_{i j}$. Therefore, the degenerate path can be expressed as a pair of time performance metrics $\left(t_{i 1}, y_{i 1}\right)$, $\left(t_{i 2}, y_{i 2}\right),\left(t_{i 3}, y_{i 3}\right), \ldots,\left(t_{i m i}, y_{i m i}\right)$, for $i=1,2, \ldots, n, m i$ represents the test time point of each unit.

$$
\begin{aligned}
& y_{i j}=D\left(t_{i j} ; \alpha ; \beta_{i}\right)+\varepsilon_{i j} \\
& D\left(t_{i j} ; \alpha ; \beta_{i}\right) \text { is the act }
\end{aligned}
$$

is the actual degraded path of unit $i$ at the measurement time $t_{i j}$. $\alpha$ is a fixed effect vector, and the fixed effect of each unit remains same. $\varepsilon_{i j}$ is a random effect vector, which varies with different units, representing the measurement error of unit $i$ at time, which is assumed to be a normal distribution with zero mean and $\delta$ variance.

The approximate method steps are as follows:

1) According to the data $\left(t_{i 1}, y_{i 1}\right),\left(t_{i 2}, y_{i 2}\right),\left(t_{i 3}\right.$, $\left.y_{i 3}\right)$...First, use the NLS method to estimate the parameters of the degraded path model (fixed effect parameters $\alpha$ and random effect parameters $\beta_{i}$ ).

2) Extrapolate the degeneration path model of each element to the critical failure threshold $D_{f}$. When $D\left(t_{i j} ; \alpha ; \beta_{i}\right)=D_{f}$, the time of "false" failure (nonactual failure) of each unit can be predicted.

3) Fit the probability distribution of these "pseudo" life data and estimate the relevant parameters of the distribution.

4) Evaluate the reliability of the sample according to the analysis reliability function $R(t)$ and MTTF.

\section{Basic theory of IES TM-21 standard}

IES TM-21 is a lumen maintenance life prediction method recommended according to the LM-80-08 report. The data used in TM-21 is the data in the LM-80-08 report, and the sample size set recommended by IES TM-21 The minimum is 20 . When the test duration $\mathrm{D}$ of the data in the report is 6000 hours to 10000 hours, the data used for curve fitting in the data set should be the last 5000 hours of data. Readings before 1000 hours are not used for curve fitting. For a data set with a test duration greater than 10,000 hours, the data after $50 \%$ of the overall measurement duration are used for curve fitting. In other words, the data between $\mathrm{D} / 2$ and $\mathrm{D}$ are all used for curve fitting. For example, if the test duration is 13000 hours, the data used should be measured between 6500 hours and 13000 hours. If there is no measurement data at point $\mathrm{D} / 2$, the data at the next lower time point should be included in the fitted data set. For example, if D is 13000 hours, data is collected every 1000 hours, and the data between 6000 hours and 13000 hours is used as the fitting data.

\section{Basic theory of IES TM-21 standard}

\subsection{NLS fitting results}

The data in this article is derived from the LM-80-08 report. The data includes 6,000-hour test points (interval time of 1,000 hours) for the lumen maintenance data's 20 units of LED luminous flux. After the initial 1,000 hours, the interval of 1,000 hours is used as a fitting data, according to the NLS operation steps shown in section 1, use the lumen maintenance data for each test time to use the NLS method to fit the LED lumen decay curve. Figure 1 is the estimated lumen life $L_{70}$ of a certain unit, and the fitted curve parameter estimation and lumen life $L_{70}$ results are shown in Table 1.

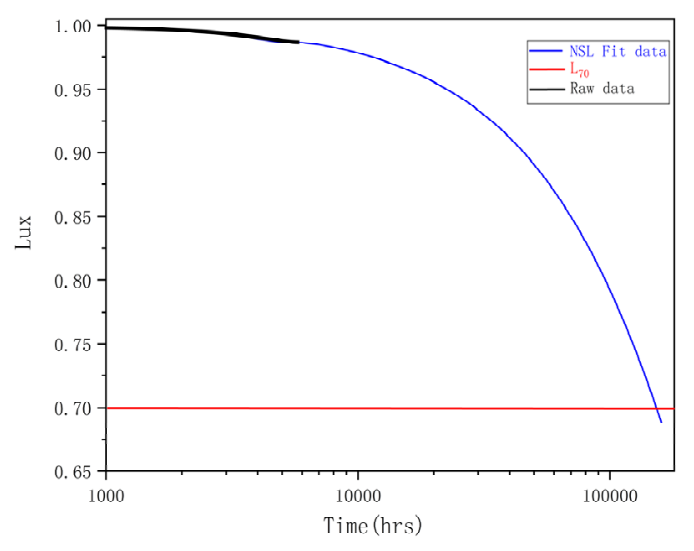

Fig 1. Lumen life $L_{70}$ test chart.

Table1. Attenuation model parameter estimation and lumen life $L 70$.

\begin{tabular}{|c|c|c|}
\hline parameter & $\alpha$ & 1.0020 \\
\hline parameter & $\beta$ & $2.9967 * \mathrm{e}-6$ \\
\hline $\begin{array}{c}\text { Lumen life } L_{70} \\
(\mathrm{hrs})\end{array}$ & $L_{70}$ & 119703.61 \\
\hline
\end{tabular}

Using the non-linear least squares method, the general degraded path model of each unit is estimated, as shown in Figure 2 (the left side is an enlargement of the right box). Then, by extrapolating the model of each unit to the critical failure threshold (light is reduced to $70 \%$ ), the "pseudo-life" is predicted, as shown in Table 2. 

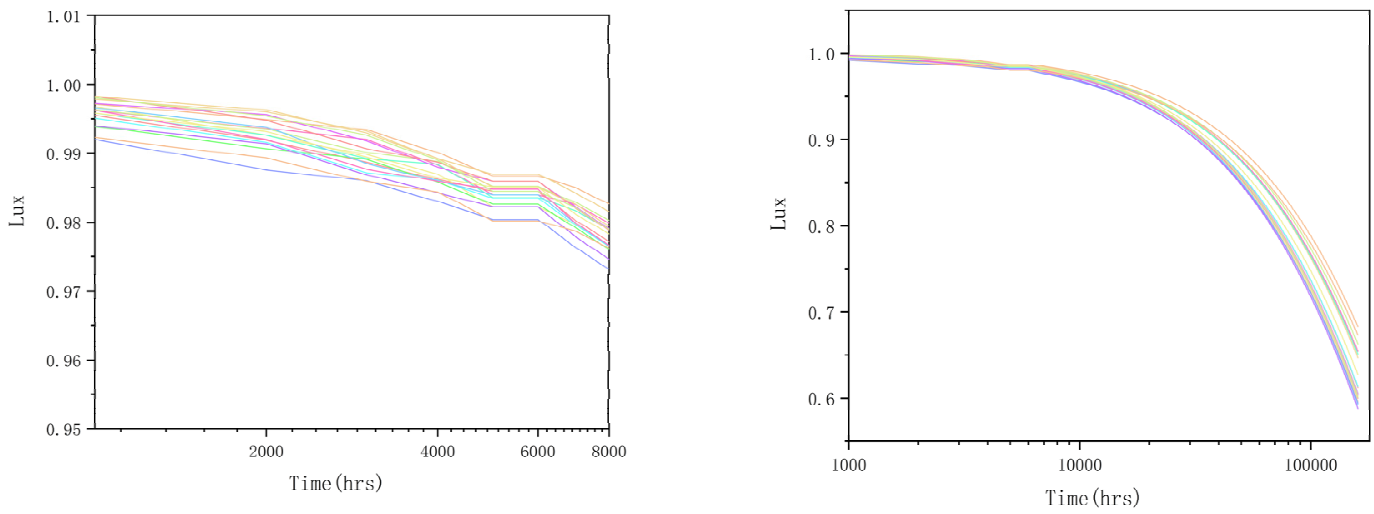

Fig 2. Data degradation path.

Table2. Degradation parameters and pseudo-lifetime of 20 units.

\begin{tabular}{|c|c|c|}
\hline Unit number & $\beta$ & Pseudo-life \\
\hline 1 & $2.34 \mathrm{E}-06$ & 152863.58 \\
\hline 2 & $2.66 \mathrm{E}-06$ & 134701.19 \\
\hline 3 & $3.08 \mathrm{E}-06$ & 116262.74 \\
\hline 4 & 2.39E-06 & 149746.29 \\
\hline 5 & $2.74 \mathrm{E}-06$ & 131375.51 \\
\hline 6 & $3.25 \mathrm{E}-06$ & 110578.73 \\
\hline 7 & $2.57 \mathrm{E}-06$ & 138901.17 \\
\hline 8 & $3.14 \mathrm{E}-06$ & 113836.32 \\
\hline 9 & $2.68 \mathrm{E}-06$ & 133108.298 \\
\hline 10 & $3.06 \mathrm{E}-06$ & 116993.78 \\
\hline Unit number & $\beta$ & Pseudo-life \\
\hline 11 & $3.29 \mathrm{E}-06$ & 109309.69 \\
\hline 12 & $3.25 \mathrm{E}-06$ & 109589.71 \\
\hline 13 & $3.32 \mathrm{E}-06$ & 107612.09 \\
\hline 14 & $3.17 \mathrm{E}-06$ & 113915.19 \\
\hline 15 & $3.19 \mathrm{E}-06$ & 112359.02 \\
\hline 16 & $3.22 \mathrm{E}-06$ & 112203.90 \\
\hline 17 & $2.45 \mathrm{E}-06$ & 144060.35 \\
\hline 18 & $3.20 \mathrm{E}-06$ & 113185.18 \\
\hline 19 & $2.92 \mathrm{E}-06$ & 122587.53 \\
\hline 20 & $3.21 \mathrm{E}-06$ & 112607.58 \\
\hline
\end{tabular}

\section{Conclusion}

LED-based lighting is one of the most influential technologies in the modern lighting era. LEDs have gradually developed into the universal lighting applications found so far, with a wide range of applications, low cost, low energy consumption, high stability and high reliability. However, so far there is no standard method that can actually measure the full life of LEDs. Therefore, how to accurately predict the lifetime of highly reliable LEDs is very important. In order to further promote the efficient and reasonable use of LEDs, this article adopts an approximate method to predict and analyse the lifetime of LED light-emitting devices. The 
approximation method first fits the failure time of each unit by using the NLS method, and then fits the failure probability distribution model to obtain the general degradation path model of the LED. Compared with the results predicted by the TM-21 standard, the approximation method can not only effectively provide more information, but also provide the reliability of the results, which will help developers to more easily decide the market application range of LEDs with different lifetimes.

\section{Acknowledgment:}

National Natural Science Foundation of China(11075026); Open Fund of Jiangxi Province Rare Earth Functional Materials Innovation Center ; Youth Jinggang Scholars Program in Jiangxi Province [2018]82, Postgraduate Innovation Special Fund Project of Jiangxi Province (YC2019-S294); Natural Science Foundation of Jiangxi Province (20192BAB206010); Youth Jinggang Scholars Program in Jiangxi Province [2018]82; Research Project of Education Department of Jiangxi Province (No. GJJ180462); Ministry of Education Science and Technology Project(3401223267).

\section{References}

1. G. Lozano, S. R. K. Rodriguez, M. A. Verschuuren, and J. G. Rivas, "Met-allic nanostructures for effificient LED lighting," Light Sci. Appl., vol. 5, p. e16080, Jun. 2016.

2. P. Lall, H. Zhang, and L. Davis, "Assessment of lumen degradation and re-maining life of LEDs using particle fifilter,' in Proc. ASME Int. Techn. Conf. Exhibit. Packag. Integr. Electron. Photon. Microsyst., 2013, pp. 1-13.

3. Approved Method for Lumen Maintenance Testing of LED Light Source, IES-LM-80-08, 2008.

4. Jiajie, Fan, Kam-Chuen, et al. Lifetime Estimation of High-Power White LED Using Degradation-DataDriven Method[J]. Device \& Materials Reliability IEEE Transactions on, 2012.

5. Fan J, Yung K C , Pecht M . Lifetime Estimation of High-Power White LED Using Degradation-DataDriven Method[J]. IEEE Transactions on Device and Materials Reliability, 2012, 12(2):470-477.

6. Fan, H. B. , et al. "An effective prediction method for LED lumen maintenance." IEEE, 2013. 